\title{
A Novel Fuzzy Clustering with Metaheuristic based Resource Provisioning Technique in Cloud Environment
}

\author{
Ahmed N. Al-Masri ${ }^{1}$, Manal Nasir ${ }^{2}$ \\ ${ }^{1}$ American University in the Emirates, Dubai, UAE \\ ${ }^{2}$ Information Technology Department, Georgia Gwinnett College, USA \\ Emails: ahmed.almasri@aue.ae, Mnasir1@ggc.edu
}

\begin{abstract}
Cloud Computing (CC) becomes a commonly available tool to enable quick, on-demand services from a shared pool of configurable computing resources which can be allocated and utilized. Resource provisioning is a major issue in $\mathrm{CC}$ environment which ensures guaranteed outcomes on the applications related to CC. This study introduces an efficient fuzzy c-means clustering (FCM) with hybrid grey wolf optimization (GWO) and differential evolution (DE) algorithm, called FCM-GWODE for resource provisioning in cloud environment. The aim of the FCM-GWODE technique is to allocate the resources in such a way that the resource utilization can be accomplished. In addition, the FCM technique with metaheuristics is applied to partition the resources and scalable searching process can be minimized. Moreover, the GWODE algorithm is derived by resolving the local optima issue of the GWO and improve the population diversity using DE. A comprehensive simulation process takes place using CloudSim tool and the results are inspected interms of several evaluation metrics. The simulation results highlighted the supremacy of the FCM-GWODE technique over the other methods.
\end{abstract}

Keywords: Resource provisioning, Cloud computing, Fuzzy clustering, Hybrid algorithms, Resource utilization, GWO algorithm.

\section{Introduction}

The conception of cloud computing (CC) clearly reflects the features of data provision in Internet phase; while the search of the vision of CC brought a novel challenge to information technology (IT) [1, 2]. Act as an important research application, the datacenter is launching a sequence of technological innovations to carry out the main characteristics of $\mathrm{CC}$, for example, elasticity of extension, massive data storage, and on-demand service. The datacenter extensively adapts virtual techniques for achieving the un-coupled mode of physical application and resource [3-5]. The application uses Virtual Machine (VM) as a package unit for sharing several physical assets with another. Henceforth the resource scheduling entity is denoted as a fine grain VM rather than coarse grains service machine. Virtual technique provide accessibility to the datacenter, however, the VM resources provision bring additional problem to an effective controlling of datacenter framework. Since the major problems for CC, resource managing aim is to protect the fundamental resources complexity and heterogeneity through adapting virtualization techniques, that make the shared resource a united massive resource. Hence, it could assurance effective resource provisioning and implement resource managing techniques and methods reasonably. Hence, achieving an efficient managing of $\mathrm{CC}$ resource is confronted by various problems that are largely displayed in 3 kinds of imbalance [6,7]. Fig. 1 illustrates the overall resource provisioning process in CC [8]. 


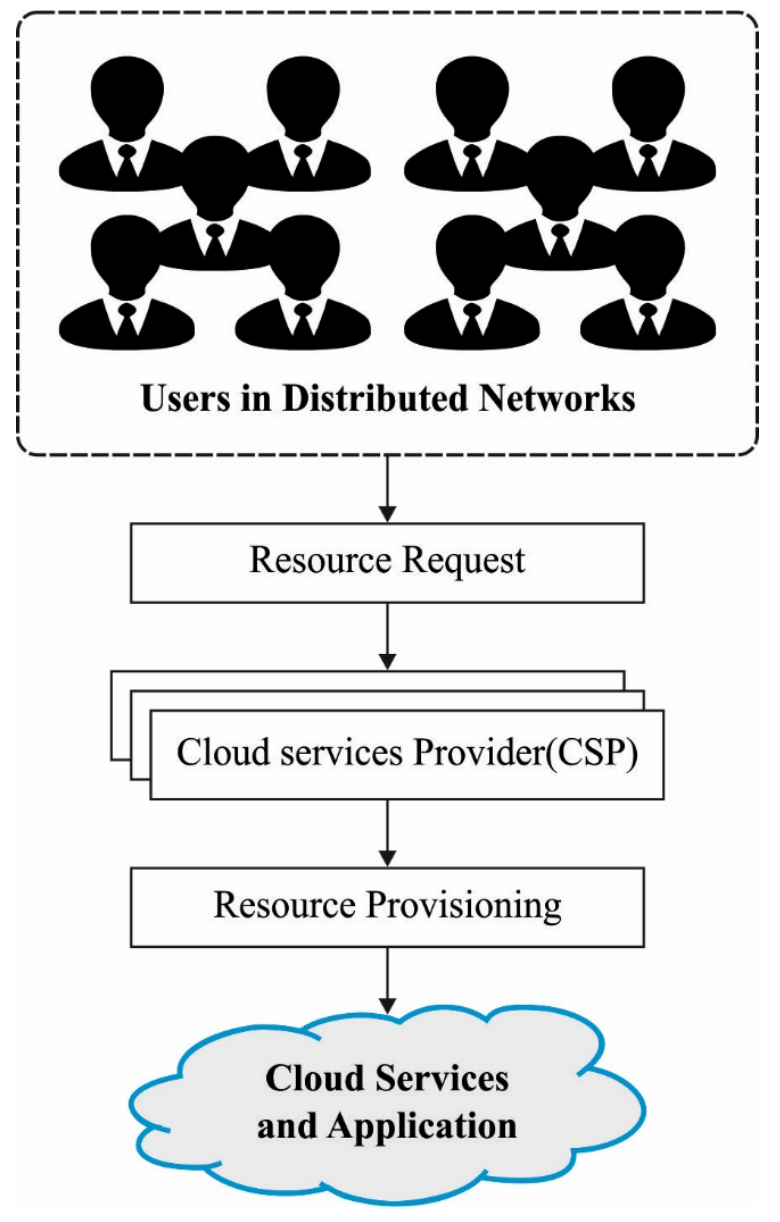

Fig. 1. Overall Resource Provisioning process in CC platform [8]

Dynamic resource management in CC platform denotes the procedure of dynamic optimization control, coordination, organization, and allocation of resources. It must assist scheduling tasks in real-time resources monitoring, job execution, and management domain/interorganizational, as well as preserve the self managing of the nearby locations, provide equivalent support of Quality of Service (QoS) $[9,10]$. It is an innovative version of resources managing, in addition, the basic element of resources managing scheme in CC platform, for shielding the complexity and heterogeneity of the fundamental resource, so as to deal with the massive distributed resource in the $\mathrm{CC}$, for control the resource efficiently, for improving resources consumption, also for providing the rational sharing of resource operations for $\mathrm{CC}$, therefore for balancing the load. As the conception of $\mathrm{CC}$ has been introducing, scheduling resources, particularly dynamic resource provision, was a significant study component. Interrelated work of resources provision is mostly from distinct points of view for constructing a $\mathrm{CC}$ method from a queue conception aims to achieve global results. But, with the aspects like incompatible interface, disparity of fundamental physical resource, and heterogeneity of built platform, experimental result demonstrates that discrepancy, hard to create similar analyses amongst one another [11-13].

[14] presented hybrid solutions for handling the resources provision problem with task analyses in a cloud platform. This solution used ICA and K-means to cluster the task presented with the end user. As well, they utilize DT model for determining scaling decisions to effective resources provision. The efficacy of the presented method in 2 actual tasks trace is assessed. [15] used to learn automated as decision makers for offloading the received active workload to the cloud/edge server. Additionally, proposed an edge servers provision method by means of LSTM method for estimating the upcoming workloads and RL methods for making suitable scaling decisions.

[16] presents a CMCDA method to select an optimal client provider pair according to reliability for resources provision in $\mathrm{CC}$ environments. The $\mathrm{CMCDA}$ models reduce the difficulty in offering the resource for the implementation of jobs and satisfy the expectation of providers and customers in $\mathrm{CC}$

Received: February 07, 2019 Accepted: May 11, 2019 
platform. Also, the method finds an optimal customer provider pair in an effective manner through evaluating the reliability value beforehand the resources provisioning. In [17], novel solutions were introduced for provisioning resources on multilayered CC applications according to MAPE-K loop. A weight ensemble predictive model is presented for estimating the resource consumption in all the CC layers. Additionally, performances of the method and a standardization method are employed for regulating the weight of the model in the presented hybrid predictive method. Further, a DT model is proposed for analyzing the position of resources for making scaling decisions.

In [18], the integration of state action learning and GA models is employed for managing CC resources. Initially, a smart agent schedules the task in the course of the learning procedure by examining the workflows. Later, all the resources are allocated to agents, and their consumption is tried to be exploited in the learning procedure of its equivalent agents. This is performed by electing a suitable group of workloads which maximize resources consumption.

This study introduces an efficient fuzzy c-means clustering (FCM) with hybrid grey wolf optimization (GWO) and differential evolution (DE) algorithm, called FCM-GWODE for resource provisioning in cloud environment. The aim of the FCM-GWODE technique is to allocate the resources in such a way that the resource utilization can be accomplished. In addition, the FCM technique with metaheuristics is applied to partition the resources and scalable searching process can be minimized. Moreover, the GWODE algorithm is derived by resolving the local optima issue of the GWO and improve the population diversity using DE. A comprehensive simulation process takes place using CloudSim tool and the results are inspected interms of several evaluation metrics.

\section{The Proposed Resource Provisioning Technique}

In $\mathrm{CC}$, the resources attribute has three groups: bandwidth, computation, and storage. Varied processes are included in numerous assets. Some kinds of computation task and computational resource is very efficient where few bandwidth task and resource is deliberated to be important. Henceforth, in order to beat the distinct requirements of the user, the resource is divided into clusters. Due to the heterogeneity and dynamicity of fog resources, it could be complex for defining the exclusive resource. Now, it is used by the FCM-GWODE method for clustering the resource according to multidimension attribute. The group of fog assets $R=\left\{r_{1}, r_{2}, r_{3}, \cdots, r_{m}\right\}$ displays $m$ fog resources node and all the nodes consist of $n$ feature. In Eq. (5), $r_{p q}$ are qth featured attributes of resource $r_{p}$.

Before fuzzy clustering, it is important to standardize the information for different processes indicator [19]. The steps to cluster fog resources were given as follows. In fog computing framework, due to the presence of different fog assets, real information might be treated in a straightforward way, since the impacts of clustering results must not be regular. Therefore, in order to solve the instantaneous effect that is created because of the translation, SD transformation should be used for the normalization of the resources matrix information.

$$
\begin{gathered}
\mathrm{r}_{\mathrm{pq}}^{\prime}=\frac{\mathrm{r}_{\mathrm{pq}}-\overline{\mathrm{r}_{\mathrm{pq}}}}{\mathrm{S}_{\mathrm{q}}} \\
\overline{\mathrm{r}}=\frac{1}{\mathrm{~m}} \sum_{\mathrm{q}=1}^{\mathrm{n}} \mathrm{r}_{\mathrm{pq}} \\
\mathrm{S}_{\mathrm{q}}=\sqrt{\frac{1}{\mathrm{~m}} \sum_{\mathrm{p}=1}^{\mathrm{n}}\left(\mathrm{r}_{\mathrm{pq}}-\overline{\mathrm{r}}\right)^{2}}
\end{gathered}
$$

Whereas maximal values of resource in qth dimensional features are $\bar{r}, \overline{r_{p q}}$ indicate the maximal values of qth certain features of resource $r_{p}$ and SD of all resources in qth dimensional features are $S_{q}$. The calculated information satisfies a standard distribution which denotes mean as 0 and $\mathrm{SD}$ as 1 . Supposedly, resource information isn't proficient in fulfilling the plan of fuzzy matrix. Henceforth, translation range was used to covert information in matrix to be achieved in the range of zero and one. 


$$
\mathrm{r}_{\mathrm{pq}}^{\prime \prime}=\frac{\mathrm{r}_{\mathrm{pq}}^{\prime}-\min \left\{\mathrm{r}_{\mathrm{pq}}^{\prime}\right\}}{\max \left\{\mathrm{r}_{\mathrm{pq}}\right\}-\min \left\{\mathrm{r}_{\mathrm{pq}}^{\prime}\right\}}
$$

Whereas, $\min \left\{\mathrm{r}_{\mathrm{pq}}^{\prime}\right\}$ display low values in $\left\{\mathrm{r}_{1 \mathrm{q}}^{\prime}, \mathrm{r}_{2 \mathrm{q}}^{\prime}, \ldots, \mathrm{r}_{\mathrm{mq}}^{\prime}\right\}$, and $\max \left\{\mathrm{r}_{\mathrm{pq}}^{\prime}\right\}$ depict high values in $\left\{\mathrm{r}_{1 \mathrm{q}}^{\prime}, \mathrm{r}_{2 \mathrm{q}}^{\prime}, \ldots, \mathrm{r}_{\mathrm{mq}}^{\prime}\right\}$.

Although the GWO technique displays capable performances in many applications, when the training sample is further, it is made up of minimal computation speed, less accuracy, and problem of local optimal [20]. Therefore, it is used by DE i.e., incorporated with GWO for enhancing the functions which relate DE to efficient search ability for improving the GWO to remove local optimal and reach the appropriate measure amongst exploration and exploitation. Likewise, selection and difference of DE model are positioned for producing early populaces which enhance diversity of populace. Primarily DE generates new populace with the procedure of populace's crossover, collection, and difference to obtain optimal solution for improvising the populace diversity. Because of its easiness, a group of skillful parameters, and strong features, DE is frequently used in numerous optimal issues by the succeeding process.

\section{Initialization Populace}

Initially, the early populace of $\mathrm{DE}$ occurs by:

$$
\left\{x_{i}(0) \mid x_{i, j}^{L} \leq x i, j(0) \leq x_{i, j}^{U}, i=1,2, \ldots, N P, j=1,2, \ldots, D\right\},
$$

Whereas $\mathrm{x}_{\mathrm{i}}(0)$ denotes the ith individuals, $\mathrm{j}$ indicates the dimensions.

$$
x i, j(0)=x_{i, j}^{L}+\operatorname{ran}(0,1)\left(x_{i, j}^{L I}-x_{i, j}^{L}\right),
$$

Whereas $\mathrm{x}_{\mathrm{i}, \mathrm{j}}^{\mathrm{LI}}$ and $\mathrm{x}_{\mathrm{i}, \mathrm{j}}^{\mathrm{L}}$ indicates the upper and lower bound of the $\mathrm{j}$ dimensions, correspondingly, ran $[0,1]$ represents a random amount in the range of zero and one.

\section{Mutation}

DE performs the understanding of an individual variation with differential mechanisms. The normal differential mechanisms randomly select a group of 2 different individuals in the populace, perform vector scaling difference, and synthesize the vector using the individual that undergoes mutation procedure.

$$
\mathrm{v}_{\mathrm{i}}(\mathrm{j}+1)=\mathrm{x}_{\mathrm{r} 1}(\mathrm{j})+\mathrm{F}\left(\mathrm{x}_{\mathrm{r} 2}(\mathrm{j})-\mathrm{x}_{\mathrm{r} 3}(\mathrm{j})\right),
$$

Whereas $r 1-r 3$ indicates random number and $F$ indicates scaling factor.

\section{Crossover}

Generally, it occurs on $j$ th populace $\left\{x_{i}(j)\right\}$ and the alternate intermediates are $\left\{v_{j}(j+1\}\right.$.

$$
u_{i, j}(j+1)= \begin{cases}v_{i, j}(j+1) & \text { if } \\ x i, j(j) & \text { otherw }(0,1) \leq C R\end{cases}
$$

Whereas CR shows likelihood crossover

\section{Selection}

Now, greedy selection processes are used in DE, showed the optimum individual is selected as a new one.

$$
x_{i}(j+1)=\left\{\begin{array}{c}
u_{i}(j+1) \text { if } f\left(u_{i}(j+1)\right) \leq f\left(x_{i}(j+1)\right) \\
x_{i}(j)
\end{array}\right.
$$




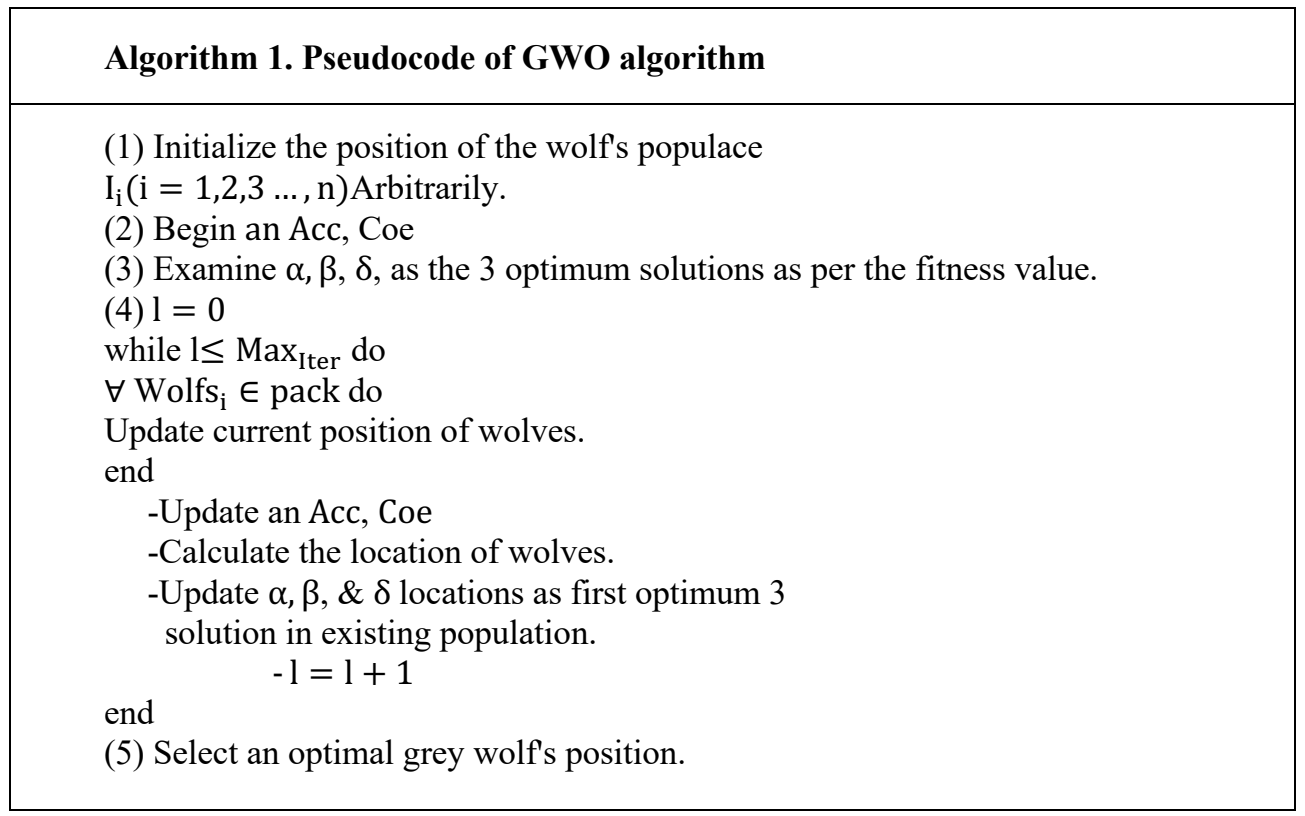

\section{Experimental Analysis}

The proposed FCM-GWODE technique is simulated using CloudSim tool. The results of the FCMGWODE technique are investigated using two benchmark datasets namely Iris and Wine datasets. Table 1 and Fig. 2 offer the result analysis of the FCM-GWODE technique on the applied test iris dataset. The results demonstrated that the FCM model has provided poor outcomes with 134 correctly clustered and 16 incorrectly clustered instances. In addition, the FAP technique has resulted in somewhat increased results with 138 correctly clustered and 12 incorrectly clustered instances. Next to that, the FCMFP technique has showcased reasonable outcomes with 142 correctly clustered and 8 incorrectly clustered instances. Finally, the FCM-GWODE technique has accomplished maximum outcome with 144 correctly clustered and 6 incorrectly clustered instances.

Table 1 Results analysis of FCM-GWODE technique on Iris Dataset

\begin{tabular}{|c|c|c|}
\hline Methods & Correctly Clustered & Incorrectly Clustered \\
\hline FCM & 134 & 16 \\
\hline FAP & 138 & 12 \\
\hline FCMFP & 142 & 8 \\
\hline FCM-GWODE & 144 & 6 \\
\hline
\end{tabular}




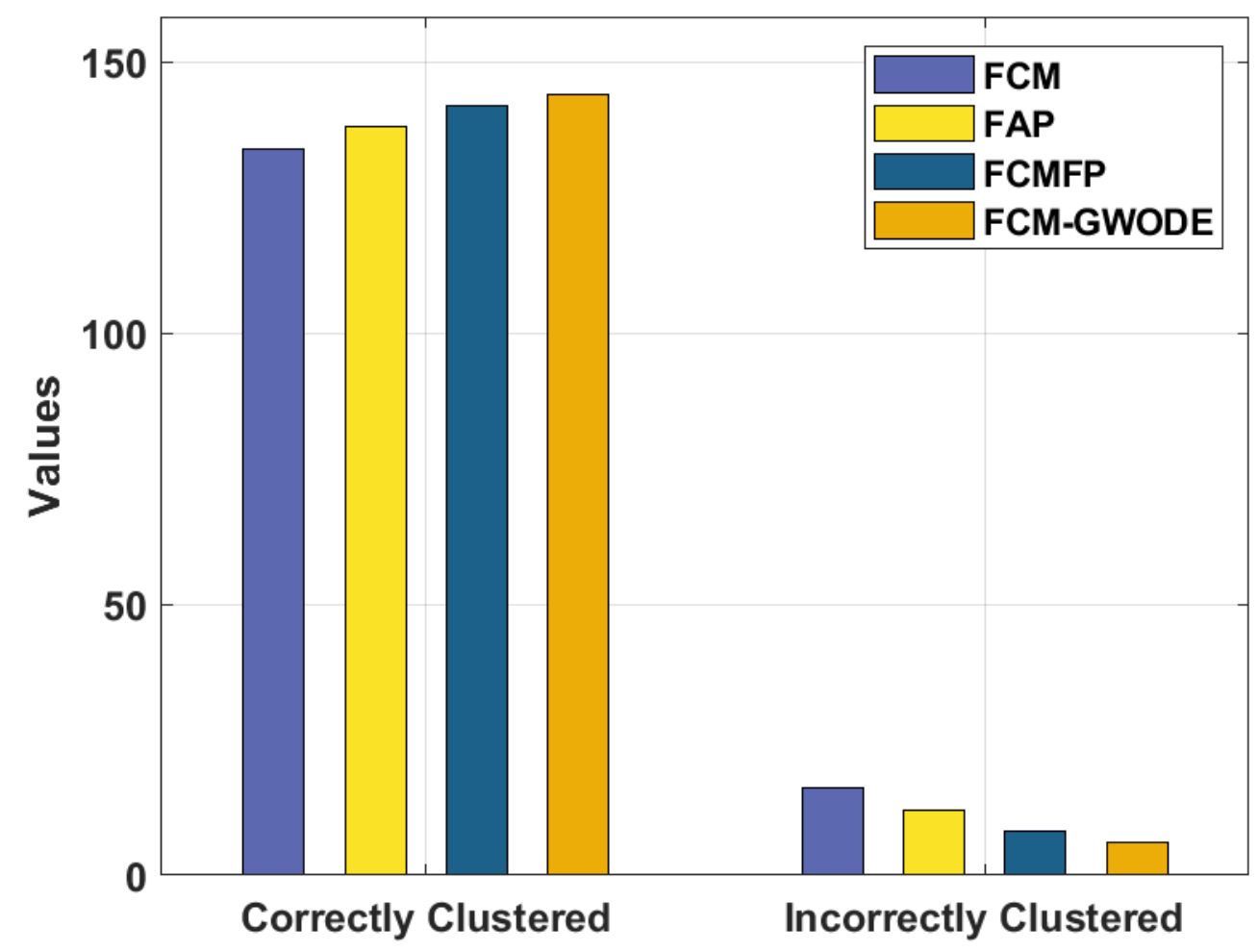

Fig. 2. Comparative study of FCM-GWODE technique on Iris dataset

Table 2 and Fig. 3 provide a comprehensive comparative study of the FCM-GWODE technique on the applied test Wine dataset. The results confirmed that the FCM model has offered lower performance with 122 correctly clustered and 56 incorrectly clustered instances. Moreover, the FAP technique has attained slightly improved results with 129 correctly clustered and 49 incorrectly clustered instances. Furthermore, the FCMFP technique has demonstrated sensible outcomes with 146 correctly clustered and 32 incorrectly clustered instances. Lastly, the FCM-GWODE technique has gained maximum outcome with 150 correctly clustered and 28 incorrectly clustered instances.

Table 2 Results analysis of FCM-GWODE technique on Wine Dataset

\begin{tabular}{|c|c|c|}
\hline Methods & Correctly Clustered & Incorrectly Clustered \\
\hline FCM & 122 & 56 \\
\hline FAP & 129 & 49 \\
\hline FCMFP & 146 & 32 \\
\hline FCM-GWODE & 150 & 28 \\
\hline
\end{tabular}




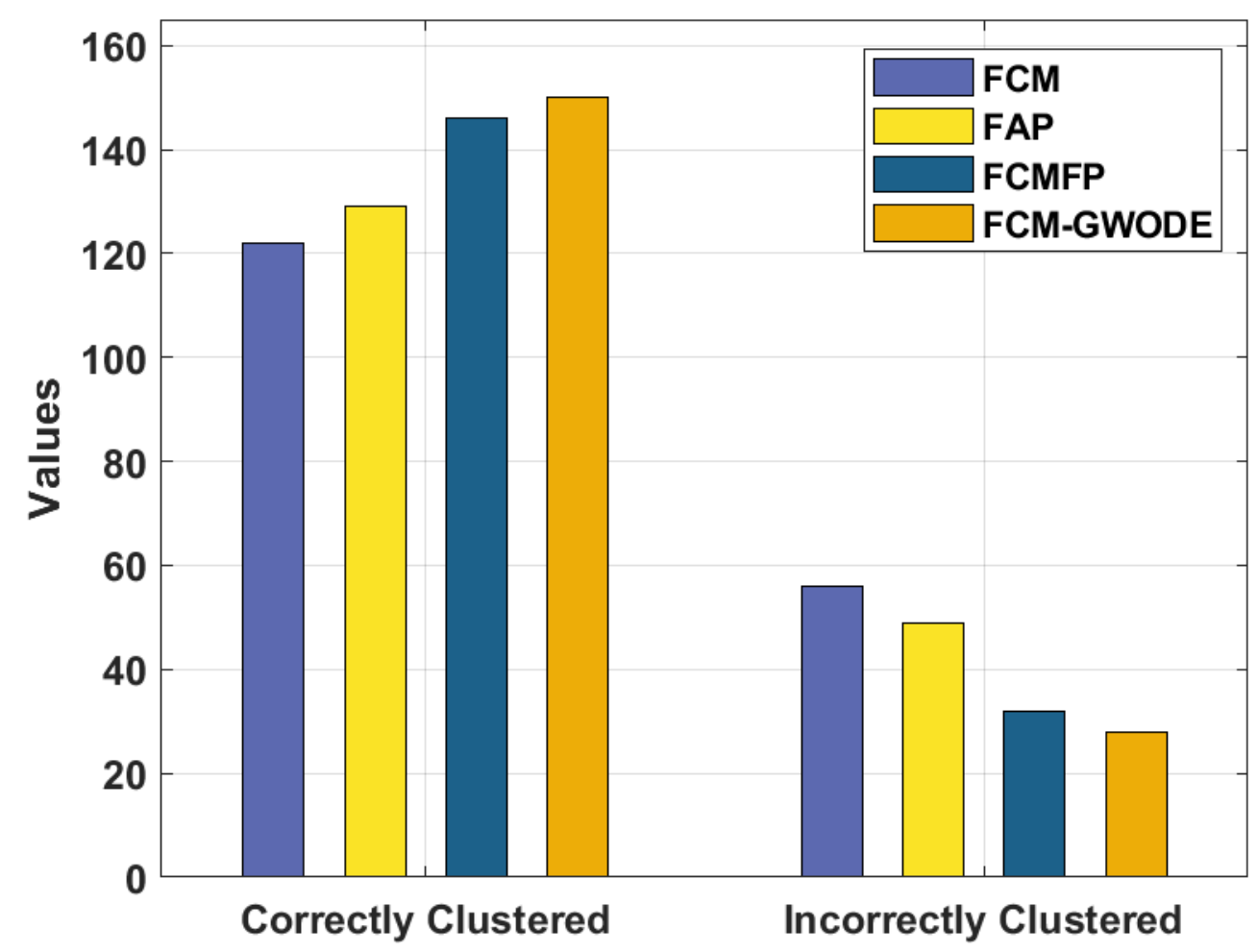

Fig. 3. Comparative study of FCM-GWODE technique on wine dataset

Detailed correct rate analysis of the FCM-GWODE technique with existing techniques is made in Table 3 and Fig. 4. From the results, it is obviously clear that the FCM-GWODE technique has resulted in maximum correct data on the applied two datasets. For instance, under Iris dataset, the FCM-GWODE technique has resulted in an increased correct rate of 0.9600 whereas the FCM, FAP, and FCMFP techniques have accomplished a reduced correct rate of $0.8933,0.9200$, and 0.9466 . Similarly, under Wine dataset, the FCM-GWODE technique has resulted in an increased correct rate of 0.8426 whereas the FCM, FAP, and FCMFP techniques have accomplished a reduced correct rate of $0.6850,0.7250$, and 0.8202. Therefore, the FCM-GWODE technique has appeared as an effective tool for provisioning resources in the cloud platform.

Table 3 Correct Rate of Existing with Proposed Method

\begin{tabular}{|c|c|c|c|c|}
\hline Methods & FCM & FAP & FCMFP & FCM-GWODE \\
\hline Iris & 0.8933 & 0.9200 & 0.9466 & 0.9600 \\
\hline Wine & 0.6850 & 0.7250 & 0.8202 & 0.8426 \\
\hline
\end{tabular}




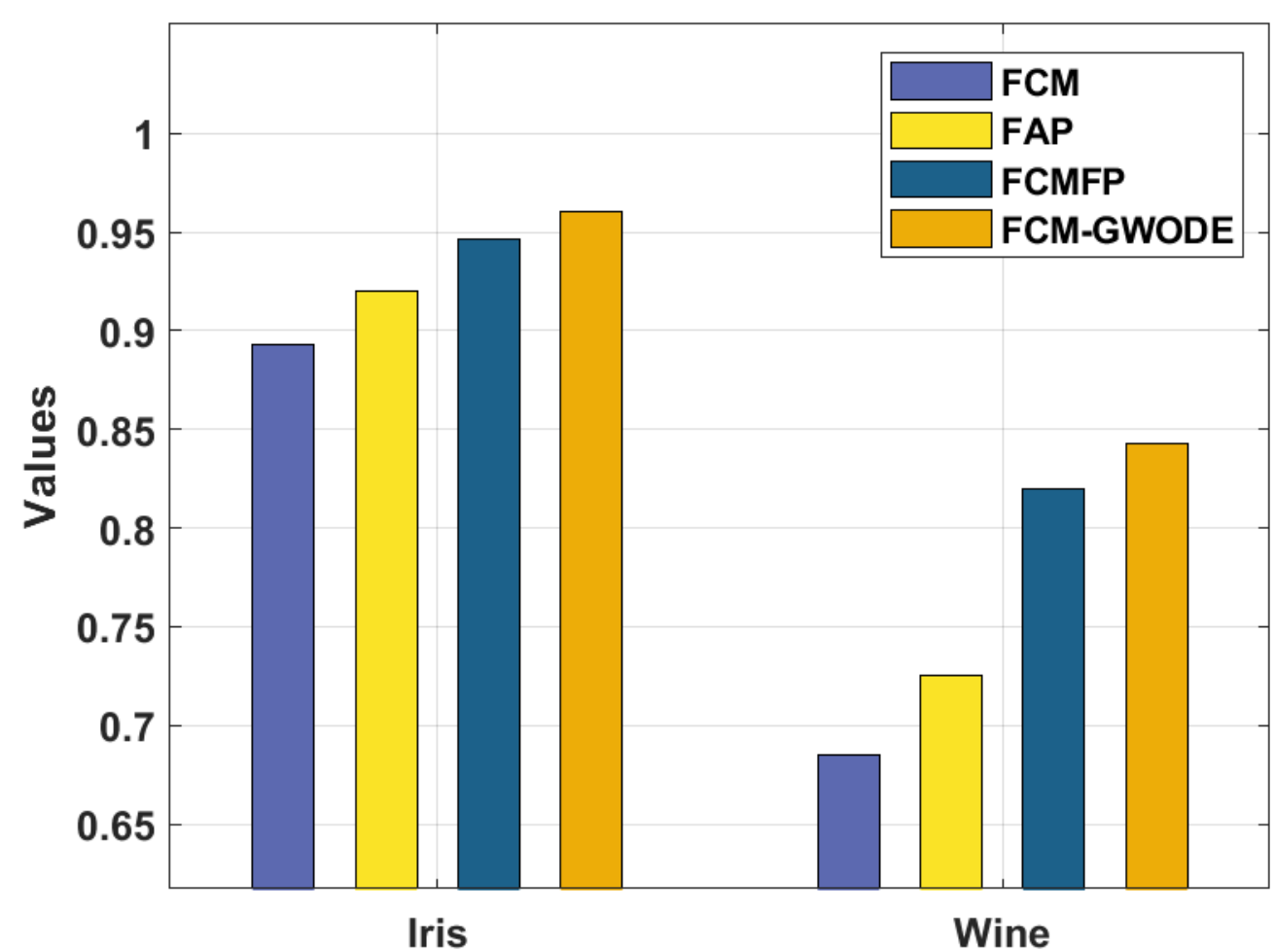

Fig. 4. Comparison of Correct Rate of Existing with Proposed Method

\section{Conclusion}

This study has introduced an efficient FCM-GWODE technique for resource provisioning in cloud environment. The FCM-GWODE technique is intended to allocate the resources in such a way that resource utilization can be accomplished. In addition, the FCM technique with metaheuristics is applied to partition the resources and scalable searching process can be minimized. Moreover, the GWODE algorithm is derived by resolving the local optima issue of the GWO and improve the population diversity using DE. A comprehensive simulation process takes place using CloudSim tool and the results are inspected interms of several evaluation metrics. The simulation results highlighted the supremacy of the FCM-GWODE technique over the other methods. In future, load balancing and task scheduling approaches can be focused to improve resource utilization in cloud platforms.

\section{References}

[1] Varshney, S., Sandhu, R. and Gupta, P.K., 2019, April. QoS based resource provisioning in cloud computing environment: a technical survey. In International conference on advances in computing and data sciences (pp. 711-723). Springer, Singapore.

[2] Ralha, C.G., Mendes, A.H., Laranjeira, L.A., Araújo, A.P. and Melo, A.C., 2019. Multiagent system for dynamic resource provisioning in cloud computing platforms. Future Generation Computer Systems, 94, pp.80-96.

[3] Shahidinejad, A., Ghobaei-Arani, M. and Masdari, M., 2021. Resource provisioning using workload clustering in cloud computing environment: a hybrid approach. Cluster Computing, 24(1), pp.319-342.

[4] Suresh, A. and Varatharajan, R., 2019. Competent resource provisioning and distribution techniques for cloud computing environment. Cluster Computing, 22(5), pp.11039-11046.

[5] Aslanpour, M.S., Dashti, S.E., Ghobaei-Arani, M. and Rahmanian, A.A., 2018. Resource provisioning for cloud applications: a 3-D, provident and flexible approach. The Journal of Supercomputing, 74(12), pp.6470-6501.

[6] Ma, X., Wang, S., Zhang, S., Yang, P., Lin, C. and Shen, X.S., 2019. Cost-efficient resource provisioning for dynamic requests in cloud assisted mobile edge computing. IEEE Transactions on Cloud Computing.

[7] Khorsand, R., Ghobaei-Arani, M. and Ramezanpour, M., 2018. FAHP approach for autonomic resource provisioning of multitier applications in cloud computing environments. Software: Practice and Experience, 48(12), pp.2147-2173. 
[8] Nigam, S. and Bajpai, A., 2017. An Optimal Resource Provisioning Algorithm for Cloud Computing Environment. Oriental Journal of Computer Science and Technology, 10(2), p.371.

[9] Xu, X., Mo, R., Dai, F., Lin, W., Wan, S. and Dou, W., 2019. Dynamic resource provisioning with fault tolerance for data-intensive meteorological workflows in cloud. IEEE Transactions on Industrial Informatics, 16(9), pp.6172-6181.

[10] Toosi, A.N., Sinnott, R.O. and Buyya, R., 2018. Resource provisioning for data-intensive applications with deadline constraints on hybrid clouds using Aneka. Future Generation Computer Systems, 79, pp.765-775.

[11] Bhardwaj, T. and Sharma, S.C., 2018. Fuzzy logic-based elasticity controller for autonomic resource provisioning in parallel scientific applications: A cloud computing perspective. Computers \& Electrical Engineering, 70, pp.1049-1073.

[12] Moreno-Vozmediano, R., Montero, R.S., Huedo, E. and Llorente, I.M., 2019. Efficient resource provisioning for elastic Cloud services based on machine learning techniques. Journal of Cloud Computing, 8(1), pp.1-18.

[13] Ghobaei-Arani, M., Jabbehdari, S. and Pourmina, M.A., 2018. An autonomic resource provisioning approach for service-based cloud applications: A hybrid approach. Future Generation Computer Systems, 78, pp.191-210.

[14] Shahidinejad, A., Ghobaei-Arani, M. and Masdari, M., 2021. Resource provisioning using workload clustering in cloud computing environment: a hybrid approach. Cluster Computing, 24(1), pp.319-342.

[15] Shahidinejad, A. and Ghobaei-Arani, M., 2020. Joint computation offloading and resource provisioning for e dge-cloud computing environment: A machine learning-based approach. Software: Practice and Experience, 50(12), pp.2212-2230.

[16] Vinothiyalakshmi, P. and Anitha, R., 2021. Efficient dynamic resource provisioning based on credibility in cloud computing. Wireless Networks, 27(3), pp.2217-2229.

[17] Mazidi, A., Mahdavi, M. and Roshanfar, F., 2021. An autonomic decision tree-based and deadlineconstraint resource provisioning in cloud applications. Concurrency and Computation: Practice and Experience, 33(10), p.e6196.

[18] Asghari, A., Sohrabi, M.K. and Yaghmaee, F., 2021. Task scheduling, resource provisioning, and load balancing on scientific workflows using parallel SARSA reinforcement learning agents and genetic algorithm. The Journal of Supercomputing, 77(3), pp.2800-2828.

[19] Porkodi, V., Singh, A.R., Sait, A.R.W., Shankar, K., Yang, E., Seo, C. and Joshi, G.P., 2020. Resource provisioning for cyber-physical-social system in cloud-fog-edge computing using optimal flower pollination algorithm. IEEE Access, 8, pp.105311-105319.

[20] Sivaram, M., Lydia, E.L., Pustokhina, I.V., Pustokhin, D.A., Elhoseny, M., Joshi, G.P. and Shankar, K., 2020. An optimal least square support vector machine based earnings prediction of blockchain financial products. IEEE Access, 8, pp.120321-120330. 